International Journal of Biomedicine | June 2021 - Volume 11, Issue Suppl_1: Abstracts from the Third Russian International Conference "Cryo-electron microscopy 2021: achievements and prospects"

POSTER ABSTRACT PRESENTATIONS

SESSION TITLE: STRUCTURE OF MEMBRANE PROTEINS

DOI: 10.21103/IJBM.11.Suppl_1.P11

\title{
Abstract P-11: Microscale Thermophoresis of Mycobacterial Cytochrome P450 with Azole Drugs
}

Ivan Kapranov $^{1}$, S. Bukhdruker ${ }^{4}$, M. Karpova ${ }^{2}$, Yulia Zagryadskaya ${ }^{1}$, Ivan Okhrimenko $^{1}$, A. Gilep ${ }^{3}$, N. Strushkevich ${ }^{2}$, Valentin Borshchevskiy ${ }^{1}$

${ }^{I}$ Moscow Institute of Physics and Technology (National Research University), Moscow, Russia

${ }^{2}$ Skolkovo Institute of Science and Technology, Moscow, Russia

${ }^{3}$ Institute of Bioorganic Chemistry of NASB, Moscow, Russia ${ }^{4}$ ESRF - The European Synchrotron, Grenoble, France

Background: Cytochrome P450 family members are found in most organisms where they are involved in the metabolism and synthesis of steroids, bile acids, unsaturated fatty acids, phenolic metabolites as well as exogenic chemicals. Drugs targeting cytochrome P450 have been shown to inhibit the growth of Mycobacterium tuberculosis, the causative agent of one of the deadliest diseases - tuberculosis. Recently, we showed that CYP124, CYP125, and CYP142 can bind and metabolize a panel of human immunoactive oxysterols in vitro (Varaksa et al., 2021) and one of them (CYP124) can metabolize antituberculosis drugs (Bukhdruker et al., 2020). Thus, inhibition of cytochrome P450 is a promising strategy for the development of new anti-tubercular drugs.

The existing methods used to assess protein-ligand interactions for cytochromes P450 (spectral titration and Surface Plasmon Resonance) have a number of limitations. In this regard, we used an alternative approach for this purposes microscale thermophoresis (MST) which was not previously used for proteins of the cytochrome P450 superfamily

Methods: Here we show that MST can be used to determine the micromolarrange dissociation constants $(\mathrm{Kd})$ of membrane-associated mycobacterial cytochrome CYP124 with small-molecule azole drugs. CYP124 was fluorescently labeled with Cy3-NHS and MST curves were collected at Monolith NT.115 instrument (blue/green channel, NanoTemper Technologies) in presence of various concentrations of azole compounds: econazole, 
ketoconazole, itraconazole, and miconazole. The experimental results were approximated by the second-order bimolecular binding equation as well as by the Hill-Langmuir equation.

Results: Therefore, MST is a valuable method for the assessment of cytochrome P450 binding to their ligands for cases when traditional approaches are not applicable. The binding regime of CYP124 with azole derivatives was characterized by the structure of the CYP124 complex with carbethoxyhexyl imidazole solved with $\sim 1 \AA$ resolution.

Key Words: tuberculosis $\bullet$ cytochrome P450 $\bullet$ microscaled thermophoresis

This work was supported by a joint grant of Belarusian Republican Foundation for Fundamental Research, B20R-061 and Russian Foundation for Basic Research, 20-54-00005

*Corresponding author: Ivan Kapranov.E-mail:kapranov.ia@phystech.edu

International Journal of Biomedicine. 2021;11 Suppl 1: S15-16.

doi: 10.21103/IJBM.11.Suppl_1.P11

(C)2021 International Medical Research and Development Corporation 